This work is licensed under a Creative Commons Attribution 4.0 International License.

Ovaj rad dostupan je za upotrebu pod licencom Creative Commons Imenovanje 4.0 međunarodna.

UDK 821.112.2.09 Hesse, H.-3

László V. SZABÓ

DOI: https://doi.org/10.29162/ANAFORA.v8i2.3

Pannonische Universität Veszprém, Ungarn

Egyetem u. 10, H-8201 Veszprém

Universität J. Selye, Slowakei

Bratislavská cesta 3322, 94501 Komárno

Origineller wissenschaftlicher Beitrag

vszabol@googlemail.com

Original Research Article

Erhalten am 16. 6. 2021

Received: 16 June 2021

Angenommen am 17. 8. 2021

Accepted: 17 August 2021

\title{
„ICH WAR ALS NEUROTIKER ERKANNT...“ KRANKHEIT, LEIDEN UND SCHMERZ BEI HERMANN HESSE
}

\section{Zusammenfassung}

Der Beitrag geht dem Themenkomplex Krankheit-Leiden-Schmerz bei Hermann Hesse nach, indem er einen Überblick über einschlägige Texte des Autors bietet. Gefragt wird nach Relationen zwischen Krankheit, Genie und Kunst in Prosatexten wie Taedium vitae, Gertrud, Kurgast oder Der Steppenwolf teils in Bezug auf Hesses Biographie, teils auf zeitgenössische Diskurse über (psychische) Krankheit oder Heilung, wie sie etwa bei Nietzsche oder Freud vorgeprägt waren. Es werden auch Verbindungslinien zwischen Hesses Auffassung der Krankheit als psychogenen Phänomens und seinem Kunstverständnis verfolgt. Schließlich werden die von Hesse etwa im Kurgast oder im Steppenwolf dargestellten psycho-physiologischen Heilungsprozesse ins Auge gefasst.

Schlüsselwörter: Krankheit, Genie, Psychoanalyse, Kunst, Poetik, Humor 


\section{Einleitung}

Krankheit, Leiden und Schmerz bilden bei Hermann Hesse einen bezugsreichen Themenkomplex, der sich in seine philosophisch-poetische Weltanschauung fußend auf Kierkegaard, Schopenhauer und Nietzsche einbetten lässt, aber auch auf psychologischen Lehren wie jenen von Sigmund Freud und Carl Gustav Jung basiert. Krankheit ist bei ihm sowohl ein körperliches als auch ein geistig-psychologisches Phänomen, das sich in diversen Formen manifestieren und nicht zuletzt eine Quelle für Kreativität und Produktivität liefern kann. Dass Hermann Hesse selbst mehrere seelische Krisen (einschließlich Selbstmordgedanken und -versuche) durchmachte und sich sogar psychoanalytischen Heilpraktiken unterzog, wurde in der Hesse-Forschung ebenso häufig hervorgehoben wie seine folgenreiche Beziehung zur Psychoanalyse. ${ }^{1}$ Es gilt als bekannt, dass Hesse um 1916 in Zürich psychoanalytisch behandelt wurde bzw. später auch mit dem berühmten Psychoanalytiker C. G. Jung selbst gemeinsame Sitzungen hatte. Gründe für eine solcherart persönliche Kontaktaufnahme mit der Psychoanalyse hatte Hesse genug: $\mathrm{Zu}$ den Erlebnissen des Krieges und dem Tod seines Vaters kamen die depressiven Nervenausbrüche seiner Frau, die ihn zu einem Sanatoriumsaufenthalt bei Luzern veranlassten, wo er dann von Joseph B. Lang, dem Schüler C. G. Jungs behandelt wurde. Während der etwa sechzig psychoanalytischen Sitzungen erwies sich Dr. Lang als ein verständnisvoller Arzt und später auch als ein lebenslanger Freund, der Hesse u. a. auf Schriften von Jung und Freud aufmerksam machte. Es geht also um einen biographischen Aspekt, auf den die Forschung häufig rekurriert, viel häufiger als bei manchen anderen Autoren der Zeit. Diese (Über-)Betonung des Biographischen bei Hesse dürfte seine triftigen Gründe haben, die nicht zuletzt im Schaffen des Autors selbst liegen: Er selbst war geneigt, eigene ${ }^{1}$ Lebenserfahrungen, einschließlich seelisch-körperlicher Krankheiten in seinen Werken zu thematisieren. Dies lässt sich von der Themenwahl bis hin zu einzelnen Figuren in seinen Werken verfolgen, die auf den Dichter selbst anspielen, also Namen wie Hermann Lauscher, Hermann Heilner, Harry Haller oder schlicht und ergreifend „Schriftsteller Hesse“.

Die Poetisierung des Biographischen lag also offenbar in Hermann Hesses Absicht; so hat er die in den psychoanalytischen Sitzungen gewonnenen Erkennt-

${ }^{1}$ Dem Thema „Hermann Hesse und die Psychoanalyse“ wurde noch im letzten Jahrhundert eine Konferenz gewidmet (Limberg 1997). 
nisse und Erfahrungen in seinen Werken der anschließenden Zeit, so in Demian, Siddhartha und DerSteppenwolf dichterisch verwertet. Zudem gehörte er zu einer Dichtergeneration nach Sigmund Freud, die über weitreichende psychologische Kenntnisse verfügte - als Beispiele könnte man Arthur Schnitzler, Franz Kafka oder Thomas Mann nennen, die sich mit der Psychoanalyse nachweislich befassten bzw. neueste psychoanalytische Erkenntnisse in diversen Formen (etwa von Essays, Tagebucheintragungen oder narrativen Modi wie dem inneren Monolog) ins eigene Schaffen integrierten. Es gab sozusagen keinen Weg mehr zurück in die Zeit vor Freud, ebenso wenig wie in die Zeit vor Nietzsche. Psychoanalyse wurde zum Bildungsgut, zu einem unumgänglichen Erkenntnisinhalt bzw. einer beliebten Erkenntnisquelle der Kunst. Zum einen wurde die Psychoanalyse zu einer Zeitmode, zum anderen kreuzten sich die Wege der Kunst und der Psychoanalyse unvermeidlich. Hesse selbst sprach 1918 im Namen einer ganzen Künstlergeneration in seinem Essay Künstler und Psychoanalyse über die Bedeutung der neuen Wissenschaft für das künstlerische Schaffen, wobei er es auch nicht versäumte, die Bedeutung von Nietzsches psychologischen Einsichten zu unterstreichen: „Allein schon die Bestätigungen und Korrekturen, welche Nietzsches psychologische Erkenntnisse und feinnervige Ahnungen erfuhren, waren uns überaus wertvoll." (Hesse, Gesammelte Werke Bd. 10 48) Freud und Nietzsche wurden zu Leitsternen für ganze Generationen von Künstlern um 1900 und danach. Lehren galten nunmehr als Erkenntnis- und Weltanschauungsmodelle, in Bezug auf die Rolle und Berufung der Kunst nicht weniger als in jenem auf Genie, Krankheit oder Leiden bzw. ihre Verflechtung.

Das Thema Genie war zwar nicht neu, es rückte ja bereits bei den Dichtern des Sturm und Drang in den Vordergrund, doch lässt sich die Idee einer Verbindung zwischen einem (krankhaften) Genie und der künstlerischen Kreativität als ein Novum in den Diskursen der Zeit, als eine moderne Idee mit großem Attraktions- und Inspirationswert konstatieren. Dabei waren die Diskurse über Genie und Krankheit vielfältig; der eine ging von einer engen Verflechtung zwischen Genialität und (geistig-psychischer) Krankheit bzw. „Wahnsinn“ aus: Man trifft auf solche in den damaligen (biographischen) Darstellungen von Nietzsches Schaffen, in der physiologisch-psychologischen Fundierung seiner epochemachenden Philosophie. Zweitens lassen sich literarische Diskurse nachzeichnen, die eine Verbindung zwischen Krankheit und Künstlertum thematisieren. Es ist keine Seltenheit in den Texten der klassischen Moderne (zu der man auch Hermann Hesse bei all seinen „romantischen“ Zügen zählen 
kann), dass Künstlerfiguren im Vordergrund stehen, deren Kreativität eine Art kompensatorische Relation mit ihrer Krankheit oder Behinderung erkennen lässt. Thomas Manns Buddenbrooks ist wohl das berühmteste Beispiel dafür, wie Kunst oder künstlerische Begabung als Symptome der Dekadenz bzw. der moralisch-kulturellen Degenereszenz gedeutet werden können.

Auch Hesse entging die mögliche Verbindung zwischen Genie und Krankheit nicht. In seinem Aufsatz Goethe und Bettina (1924) brachte er das Problem des gespannten Verhältnisses zwischen Genie und seiner Umwelt auf den Punkt:

Das Genie, wo es auch auftaucht, wird entweder von der Umgebung erdrosselt oder tyrannisiert sie; es gilt ohne Widerspruch als die Blüte der Menschheit und richtet doch überall Not und Wirrnis an, es tritt stets vereinzelt auf, zur Einsamkeit verurteilt, ist unverderblich und hat stets eine Tendenz zur Selbstaufgabe. (Hesse, Gesammelte Werke Bd. 12 194)

Doch werden die Selbstaufgabe und Selbstverneinung des Genies von Hesse nicht nur als eine geistesgeschichtliche, sondern auch als eine biologische Angelegenheit verstanden. So meint er in einem Essay über Novalis vom selben Jahr: „Genie [ist] nicht nur eine geistesgeschichtliche, sondern ebenso, ja vor allem, eine biologische Angelegenheit [...]. In der neueren deutschen Geistesgeschichte sind die edelsten Gestalten dieser Art Hölderlin, Novalis und Nietzsche." (234) Diese „edelsten Gestalten“ zeigen ein biologisch schwaches Leben, das mit der Lebensbejahung in ihren Werken in Gegensatz steht. Hervorgehoben wird also von Hesse nicht, wie bei Thomas Mann oder bei anderen Nietzsche-Rezipienten (V. Szabó 2007), die pathologische, sondern vielmehr die biologische Beschaffenheit des Genies, dessen tragisches Geschick nicht nur im Gemüt, sondern auch in der Zeit geschrieben steht. Das Genie ist nicht bloß eine pathologische Erscheinung, sondern eher ein sensibler Seismograph seiner Zeit. Der Akzent wird damit von der Abstempelung des Genies als einer kranken Seele auf die Verantwortung der Mitwelt verlegt, die das Genie in die Krankheit treibt. Das Genie ist nicht ab ovo ein Psychopath, kann aber unter ungünstigen Zeit- und Kulturumständen einer werden. Gleichzeitig lässt sich bei Hesse, wohl in Anlehnung an Nietzsche, eine Aufwertung der Genies, der „wahrhaft Selbstsüchtigen“ bemerken, denen er, trotz ihrer Neigung zur Krankheit und Leidensfähigkeit, die lebendige, ja frohe Seite der Welt zuspricht: 
Wenn jemals die Welt durch Menschen verbessert, durch Menschen reicher geworden, lebendiger, froher, gefährlicher, lustiger geworden ist, so ist sie es nicht durch Verbesserer geworden, sondern durch jene wahrhaft Selbstsüchtigen [...] welche kein Ziel kennen, welche keine Zwecke haben, denen es genügt, zu leben und sich selbst zu sein. Sie leiden viel, aber sie leiden gerne. Sie sind gerne krank, wenn es ihre Krankheit ist, die sie leiden sollen, ihre wohlerworbene, eigene, eigenste. Sie sterben gerne, wenn es ihr Tod ist, den sie sterben dürfen, ihr wohlerworbener, eigener Tod! (Hesse, Gesammelte Werke Bd. 10 490)

Ganz im Sinne Nietzsches vollzieht hier Hesse gleichsam eine Heroisierung der Leidensfähigkeit und sogar der Krankhaftigkeit von genialen, „, selbstsüchtigen“ Individuen, denen er die Bereitschaft, den „eigenen Tod“ zu sterben, hochrechnet. Krankheit und Leiden sind also für Hesse nicht bloß Dekadenzsymptome, sondern eher das Zeichen einer höheren Berufung, eines besonderen, differenzierten Individuums (V. Szabó 2012), das der Welt keinen Zwang antut, um sie zu „verbessern“, sondern in und durch sich selbst ein Modell höherer Menschlichkeit darstellt.

\section{Poetik der Krankheit und des Leidens}

Bei allem Respekt Hesses für die Genies als besonders humane und kreative Individuen, sind doch nicht all seine Figuren gleich Genies. Während er sich in den Essays und Briefen häufiger mit der Genie-Problematik auseinandersetzt und damit einen verbreiteten Diskurs seiner Zeit aufgreift, fällt in seinen fiktiven Texten das wiederholte Vorkommen von kranken, krankhaften oder auch behinderten Figuren auf. Man könnte sie allesamt als Dekadenzerscheinungen einer Fin-de-siècle-Stimmung abtun, aber damit würde man bei Hesse wohl zu kurz greifen. Vielmehr lassen sie sich mit einer Art Obsession Hesses mit dem Phänomen der Krankheit in Verbindung bringen, deren Wurzeln, wie bereits angedeutet, im Biographischen liegen mögen. Es geht allerdings bei all den Figuren seiner Erzählungen und Romane vor allem um geistig-seelische Krankheiten bzw. um solche des Gemüts, wie es noch in der Romantik hieß, zu der Hesses Texte vielfache Bezüge aufweisen. Figuren wie Klein oder Harry Haller zeigen eine Vielfalt von Symptomen vom Lebensüberdruss zu Depression, von Pessimismus zu suizidalen Neigungen. Sie leiden an einer „Krankheit zum Tode“ im Kiergaard'schen Sinn und sind von der Sinnlosigkeit des Daseins 
überzeugt. Manche von ihnen gehen daran zugrunde, andere aber finden Auswege aus dem deprimierenden Zustand des Lebensekels.

Für letzteren hatte bereits Seneca den Ausdruck taedium vitae gefunden, den Hesse zum Titel einer seiner frühen Erzählungen machte. Die melancholisch-bedrückende Grundstimmung der Erzählung wird durch die Zeitangabe „Anfang Dezember“ (Hesse, Meistererzählungen 65) bereits in der Exposition nur verstärkt. In der Einförmigkeit des Alltags scheint kein Ausweg aus der Melancholie und dem Ekel am Leben möglich. Lebensüberdruss, chronische Schlaflosigkeit, das Gefühl der Sinnlosigkeit und des „Totseins“ scheinen unüberwindbar. Keine Nacht, kein Tag unterscheidet sich von den anderen, ihre horis successivis lassen nur die erschütternde Erkenntnis zu: „Sie haben keinen Sinn.“ (66) Den einzigen Trost bieten die stummen Bücher, darunter eines über die theosophische Geheimlehre der Madame Blavatsky, das die Ich-Figur, wie man am Ende erfährt, von einem Antiquar gekauft hatte, um zumindest ihm, dem Buchhändler, eine Freude zu bereiten. Doch selbst dieses vermeintliche Antidot gegen das taedium vitae erweist sich schließlich als unwirksam: Bücher, selbst orientalischen Inhalts, die Hesse so häufig und gerne las, können an sich kein krankes Gemüt heilen, man muss offenbar woanders nach Genesung suchen. Die Antwort, inwiefern und wie man sich vom Lebensüberdruss heilen kann, bleibt aber Hesse in Taedium vitae ebenso schuld, wie in anderen Erzählungen seiner frühen Schaffensphase.

Die Titelfigur der anderen Erzählung, Walter Kömpff, leidet an einer Abschwächung des Willens und des Glaubens an einen Sinn des Lebens überhaupt. Langsam, aber sicher überkommt ihn das Gefühl der „Leere“, die sich seines Denkens und Empfindens unaufhaltsam bemächtigt - ein Vorgang vergleichbar von innen mit der Verbreitung einer Krankheit im Körper oder im Gehirn, von außen mit der Krankheit der Seele. Es liegt etwas Paradoxes in dieser Doppelbödigkeit der Krankheit, die den Körper und die Seele gleichzeitig ergreift: Als ob sie die zwei untrennbaren Seiten der „Medaille“ Mensch wären. Leidet die Seele, so wird früher oder später auch der Körper in Mitleidenschaft gezogen, während umgekehrt, eine starke Seele auch den Körper widerstandsfähiger machen kann. Der psychisch Leidende wird der eigenen Seelenkrankheit selten gewahr und „produziert“ Überzeugungen und „Wahrheiten“, von denen er sich schwer abbringen lässt. Er erlebt seinen eigenen Seelenzustand gleichsam als eine Offenbarung, die in seiner subjektiven Wahrnehmung keineswegs hinter, ja sogar über den Wahrheiten anderer steht. Die Einsamkeit verstärkt den Leidenden 
in seinen Überzeugungen, in solchen etwa, dass das Leben keinen Sinn habe oder des Erlebens nicht wert sei. Walter Kömpff ist einsam, wie manch andere Gestalten Hesses und der literarischen Moderne überhaupt, und Einsamkeit ist zerstörerisch, ja, sie selbst ist wohl eine Form der Krankheit. Ein Gesellschaftsleben, wie dem auch sei, wird Kömpff nicht gegönnt, so dass sein Untergang nicht aufzuhalten ist: Sein Selbstmord hat am Ende nichts Heroisches in sich, er ist das Ergebnis einer fatalen psychischen Entwicklung bzw. einer seelischen Schwäche, die der für die Moderne symptomatische Vereinsamung nichts zu widersetzen hat.

Auf ähnliche Weise leidet die Figur namens Klein in Hesses Erzählung Klein und Wagner an einem „würgende[n] Angstgefühl“, zu dem sich „Schlaflosigkeit, Depression und Alpdruck“ (Hesse, Gesammelte Werke Bd. 5 262-63) gesellen. Die Seelenkrankheit, der sich aber auch ekstatische Momente anschließen - die paradox anmuten würden, wüsste man nicht, dass Depression auch extrem heitere Stimmungswellen produzieren kann - erweist sich hier als unheilbar und führt zu einer Selbstaufzehrung, die in den Tod mündet. Selbst die Resignationslehre Schopenhauers, der in der Erzählung explizit zitiert wird, bietet keine Hilfe mehr für den sich bis zur Verzweiflung deprimierten Klein (V. Szabó 2013), der bei einer einsamen Bootsfahrt schließlich seinen - man könnte auch sagen: selbstgesuchten, selbstgewählten - Tod findet. Überhaupt lässt sich angesichts solcher Figuren die Frage stellen, woher überhaupt ihre Krankheit kommt, die bei Hesse vor allem psychischer bzw. psychogener Natur ist. Allerdings zeigen diese Figuren eine Art Grundempfindlichkeit bzw. eine Überspannung, die sich z. T. mit der Sensibilität von Hesse selbst erklären ließen, von der er bereits in der Pubertät ein spektakuläres Zeugnis ablegte, als er mit fünfzehn Jahren einen Selbstmordversuch unternahm. In den Texten der sog. klassischen Moderne zeigen zahlreiche literarische Figuren einen sensiblen Charakter, deren Schicksal in Tod oder Selbstmord als (er-)lösende Katastrophe endet. Man wäre also geneigt zu behaupten, dass (seelische) Krankheit zum Geist der modernen Zeit gehört; sie ist eine Reaktion auf eine Welt in Krise und auf eine Kultur, die im Sinne Sigmund Freuds - viel Unbehagen bereitete. Seit Nietzsche schien die Krankheit eine Art Mode geworden zu sein, ein beliebtes Thema bei mehreren Autoren, unter denen Hesse mit einer ganzen Reihe von Texten über Krankheit und Tod auf sich aufmerksam machte. Hesse war ein Stimmungskünstler, sehr empfindlich für das Krankhafte, Bedrückende und Bedrohliche in der Welt. Ja, er schien der Überzeugung zu sein, dass die ganze moderne Welt, die westliche 
Zivilisation allesamt krank sei, wofür er auch in den zwei Weltkriegen Bestätigungen fand.

Gleichzeitig stellt sich für ihn die bereits bei Nietzsche intensiv diskutierte grundsätzliche Frage nach dem Verhältnis zwischen Krankheit oder Behinderung und künstlerischer Kreativität. Im Roman Gertrud tritt Kunst in ein reziprokes Verhältnis zur Krankheit und Behinderung bzw. überhaupt zum ,Leben: Das Schaffen erscheint als eine Kompensation physisch-seelischer Behinderung, mehr noch: Letzteres erweist sich als eine notwendige Quelle für die künstlerische Kreativität bzw. den Erfolg des Künstlers. Bereits in Rosshalde erschien das scheinbar paradoxe Verhältnis zwischen einem Defizit des Lebens und der wachsenden Qualität künstlerischer Produktion wie selbstverständlich: Je tiefer der Maler Veraguth in einer Ehekrise steckt, desto wertvoller und erfolgreicher werden seine Gemälde. Seelisches Leiden scheint mitunter unabdingbar für eine höhere künstlerische Leistung, indem Kunst gleichsam Asyl in einem gescheiterten Leben bietet. ${ }^{2}$ Der Komponist Kuhn in Gertrud leidet neben seiner physischen Behinderung auch an einer Gemütskrankheit, die ihm „das Ganze falsch und dumm“ (Hesse, Gesammelte Werke Bd. 3 125) escheinen lässt. Außer der Kunst zeigt sich jedes Heilmittel unwirksam, darunter auch orientalische Lehren: Wie in Taedium vitae, so bleibt auch hier der Protagonist unbeeindruckt von der (damals modischen) Theosophie. Dies erscheint in Gertrud in der Gestalt des Theosophen Lohe, der Kuhn empfiehlt, weniger an sich als an andere zu denken, vom eigenen Leben und Leiden gleichsam abzusehen, sich selbst aus einer höheren Perspektive zu betrachten. Zudem stellt Lohe die Diagnose einer moral insanity auf, die er mit einem für den modernen Menschen charakteristischen Individualismus und einer eingebildeten Einsamkeit erklärt. Damit entpuppt sich die individuelle Krankheit als ein Abdruck der Zeit in der Seele, während die moderne Welt mit dem empfindenden, leidenden Subjekt in eine enge Relation gestellt wird. So zeigen sich Einsamkeit und Krankheit als Grundempfinden der Moderne, das die Psyche des Subjekts besonders empfindlich und anfällig macht für die kleinsten Enttäuschungen. Die andauernde Überspanntheit der Seele kann dann zu einer chronischen Gereiztheit, ggf. zu Neurosen und Depressionen führen, die aber letztendlich als Zeitkrankheiten zu Tage treten. Auch die Einbindung des Subjekts in die Gesellschaft erleidet Erschütterungen, wodurch es immer bedrohlicher von seiner Einsamkeit „um-

${ }^{2}$ Hesse selbst hat mehrere Aquarelle produziert, in deren therapeutischer Funktion er auch von Carl Gustav Jung bestätigt wurde. 
schlungen" wird, während die Selbsthilfe in eine immer unerreichbarere Ferne rückt. Das erkrankte Subjekt sieht immer ohnmächtiger den zerstörten Beziehungen zu seiner Mitwelt zu. Gleichzeitig wirkt die Depression nach innen nicht so sehr als eine Krankheit, als das verstärkte Gefühl, das Dasein überhaupt habe seinen Sinn und Zweck verloren: „Damit war der Sinn des Daseins verloren, und in seiner kranken Seele trieben zwar noch schillernde Blasen und Traumgebilde, aber keine lebendigen Keime mehr. Sein Licht war ausgebrannt, und es erlosch schnell und traurig." (257)

Einen Tiefpunkt erreicht Kuhn an einem Februartag, als er, geplagt durch „Wintermüdigkeit“, seines Außenseitertums in Liebesbeziehungen gewahr wird. Ein Kuvert mit Gertruds Handschrift auf einem Tisch reicht, um ihn in die tiefste Verzweiflung zu stoßen. Von hier bis zum Entschluss, dem eigenen Leben ein Ende zu setzen, wäre es nur ein kurzer Schritt:

Mehrere Tage kam ich weder zu Tränen noch zu Schmerzen. Ohne zu denken, hatte ich beschlossen, nicht weiterzuleben. Vielmehr hatte der Lebenswille in mir sich niedergelegt und schien verschwunden. Ich bedachte das Sterben wie ein Geschäft, das unweigerlich getan werden muß und bei dem man sich nicht besinnt, ob es angenehm ist oder nicht. (114)

Der Selbstmord wird hier zwar nicht durchgeführt, da sich Kunst bzw. das Schaffen (von Opern) als Rettung darbietet, dennoch bleibt festzuhalten, dass Hesse auch in Gertrud den psychischen Zustand des Protagonisten, die Verzweiflung und die Depression, die heftigen Gefühlsausbrüche und Stimmungswechsel, die zerreißenden Schmerzen, und nicht zuletzt auch die psychischen Phänomene der Apathie und des geschwächten Lebenswillens mit viel Können und Empathie schildert. Die Gleichgültigkeit gegen das eigene Leben lässt das Sterben als ein bloßes „Geschäft", als letzte Abrechnung mit dem eigenen Dasein erscheinen, hinter der „eisigen Hülle“ der Seele tut sich die „Schrecklichkeit des Lebens“ (116) auf, bei deren furchtbarem Anblick sogar der Tod mildere Züge annimmt. Wenn Depression künstlerisch darstellbar ist, dann gehören Hesses Werke diesbezüglich zu den feinsten.

\section{Strategien der Heilung}

Diskurse über psychische Krankheiten lassen sich also bei Hesse mehrfach führen, sind sie doch häufig rekurrierende Themen seiner Erzählungen und 
Romane. Parallel dazu werden aber auch Heilwege und -prozesse in machen seiner Werke angedeutet oder gar ausführlich entfaltet. Haben Texte wie Walter Kömpff oder Gertrud stellenweise einen durchaus depressiven Charakter, so wundert es fast, dass Hesse auch solche serenen Texte produzierte wie die „indische Dichtung“ Siddhartha (1922). Darin ist zwar Krankheit kein Thema, dennoch deutet sie auf eine Weltanschauung hin, die gegenüber dem kranken Okzident im Orient geradezu eine heile Welt sieht. Hier schien Hesse selbst seine psychischen Krisen hinter sich gelassen und in einer meisterlich poetisierten östlichen Weisheit endgültig seine Seelenruhe gefunden zu haben. Bezeichnend für diese Serenität - eher untypisch in den frühen Werken - ist die Erzählung Kurgast (1925), die deutliche autobiographische Züge zeigt, basiert sie doch auf eigenen Erfahrungen des Autors anlässlich der Kuraufenthalte in Baden (in der Schweiz) im Frühling und Herbst 1923. Auch die - diesmal vor allem - physischen Krankheiten, die im Text angesprochen werden, wie Rheuma, Gicht, Ischias oder Stoffwechselkrankheit sind womöglich biographischer Herkunft. Zudem wird der mit dem Ich-Erzähler identifizierte Kurgast, der „Schriftsteller Hesse“ vom behandelnden Arzt als „Neurotiker erkannt“ (Hesse, Gesammelte Werke $B d .7$ 22), was wiederum auf den von Hesse häufig betonten Zusammenhang zwischen der Befindlichkeit des Körpers und der Seele verweist. Die physischen Symptome bereiten zwar Missmut, Ärger, auch Gereiztheit, doch führen sie diesmal zu keiner unheilbaren Depression, denn Hesse hatte zu dieser Zeit noch ein poetisches Ass in der Hand, nämlich den Humor.

Der Humor, der also bei Hesse, bei all der Prägnanz depressiver Schilderung durchaus vorhanden ist, ${ }^{3}$ bietet sich hier als eine Möglichkeit zur Überwindung der Despression, der körperlich-seelischen Schwäche, und schließlich sogar der physischen Krankheiten an. Die Kunst ist hier zwar keine Garantie für die Heilung, dafür aber erweisen sich der Humor, das Lachen, die heitere Betrachtung der Welt um sich herum als durchaus heilsam. Das körperliche Befinden wird dabei, in dem Dialog mit dem Arzt, in Einklang übrigens mit späteren Beobachtungen über psychosomatische Krankheiten, nicht als Ursache, sondern eben als Wirkung geistig-psychischer Störungen denunziert. Jede Krankheit ist psychogener Natur, ihre Wurzeln können deshalb in dem jeweiligen seelischen $\mathrm{Zu}$ stand vermutet werden, und solange da Pessimismus, Depression und Neurose vorherrschen, kann man wenig Hoffnung auf Besserung hegen. Die Heilwirkung des Humors zeigt sich nun darin, dass er Hemmungen, Ängste, Befürch- 
tungen, ja Störungen der Psyche auflöst, neurotische Anspannungen lindert, wodurch das verhaltene, verdrängte Leben gleichsam seine Fesseln bricht. Hier scheint Hesse mehr denn je dem Ratschlag von Nietzsches Zarathustra zu folgen: „Vergesst mir das gute Lachen nicht!“

Hesses Humor, gepaart mit Ironie, auch Selbstironie, erscheint in der Sprache bzw. in der Komik der geschilderten Situationen. Die Kurgäste, heißt es, verbleiben bei einer „Zweiseitigen, bipolaren Denkart“ und einem daraus resultierenden Leiden, das ebenso wenig heroischer Natur ist, wie ihr „Schicksal“, das somit in einer ironischen Opposition steht mit dem Schicksalsbegriff eines Dostojewski oder Nietzsche. Die an Rheuma oder Gicht leidenden Kurgäste, darunter der Ich-Erzähler selbst, fixieren ihre Optik gleichsam auf die Schattenseite des Lebens, die sie mit einer Art Wahrheitsphilosophie identifizieren. So charakterisiert sich auch der Kurgast Hesse:

Ich mache indessen ruhig vom Gewohnheitsrecht aller Menschen, auch der Psychologen, Gebrauch und projiziere nicht nur in die Menschen, sondern sogar in die Dinge und Einrichtungen meiner Umgebung, ja in die ganze Welt meine Denkart, mein Temperament, meine Freuden und Leiden hinein. Meine Gedanken und Gefühle für ,richtig, für berechtigt zu halten, diesen Genuß lasse ich mir nicht rauben, obwohl die Umwelt mich stündlich vom Gegenteil zu überzeugen sucht, ja, ich mache mir nichts daraus, die Majorität gegen mich zu haben, ich gebe eher ihr unrecht als mir. (8)

Die depressive Denkart, deren Recht der "Schriftsteller Hesse“ sich vorbehält, indem er sie auf die Umwelt projiziert, sieht in der Welt mehr Grund zum Leiden als zur Freude, wobei er sein einziges Vergnügen ironischerweise im Mitleid, seine einzige „wahre Lust“ in der „Leidensgenossenschaft“ findet (11). Die skeptisch-depressive Weltsicht verdunkelt sogar scheinbar neutrale äußere Phänomene wie der Regen:

Ja, ebenso wie Schicksal und Gemüt Namen eines Begriffes waren, ebenso wie ich meinen Namen und Stand, mein Alter, mein Gesicht, meine Ischias in gewissem Sinne mir selbst erwählt und erschaffen hatte und niemand als mich dafür verantwortlich machen durfte, ebenso stand es wohl auch mit diesem Regen. Ich war bereit, ihn auf mich zu nehmen. (15) 
Humor mischt sich in den Pessimismus bei der Wahl des Zimmers, die zum Ärger mit dem holländischen Zimmernachbarn führt. Der Erzähler klagt jede Illusion der menschlichen Willensfreiheit an und plädiert im Geiste Schopenhauers für den Verzicht auf jeden Willen, der das Schicksal lenken und den Zufall austilgen zu können wähnt: „Wissen wir nicht, daß unser Schicksal uns eingeboren und unentrinnbar ist, und hängen wir nicht dennoch alle innig und glühend an der Illusion der Wahl, der Willensfreiheit?" (17) Er betrachtet die ganze Handlung und damit die erzählte Geschichte aus einer ironischen Distanz, wobei die Überzeugung von der Unbeeinflussbarkeit des Schicksals und der „drolligen Sinnlosigkeit“ jedweden Tuns die Oberhand gewinnt. Das Gefühl der Sinnlosigkeit jeder Bemühung wird nur verstärkt durch die Monotonie des täglichen Vegetierens, wo ein Morgen ebenso lästig ist wie der andere. Der Abendmensch Hesse empfindet den tiefsten Pessimismus am Morgen, wo „alle Gnade, aller Zauber, alle Musik“ weit entfernt von dem noch Schläfrigen stehen: Denn was kann man von einem Tag erwarten, der damit beginnt, dass der Mensch aufstehen muss? Die chronische Langeweile entpuppt sich zugleich als eine Erscheinung des Geistes, der mit seinen Antinomien und Widersprüchen kein Leiden und keinen Pessimismus zu beseitigen vermag: „Es gehört [...] zur Gemütsart und Denkweise des nicht mehr jungen Rheumatikers und Gichtbrüchigen, daß er die Unmöglichkeit eingesehen hat, die Welt geradlinig zu verstehen, daß er Sinn und Achtung hat für die Antinomien, für die Notwendigkeit der Gegensätze und Widersprüche.“ (33) Diese Gegensätze wurzeln im Gemüt des Menschen, der kein vollkommen logisches, kalkulierbares Wesen ist, sondern im Irrationalismus seiner eigenen Widersprüche lebt. Zu solchen irrationalen Phänomenen gehört eben die Krankheit oder der (starke oder schwache) Wille. Sie hängen auf eine geheimnisvolle, psychogene Weise zusammen, indem die Stärke des Letzteren die Erstere besiegen kann, während die Willensschwäche die Chancen der Krankheit vergrößert. Die Kurgäste werden als willensschwach entlarvt, die ihre Krankheit nicht bewältigen wollen und damit das irrationale Element in der Welt bilden, die ihren Bequemlichkeitsbedürfnissen mit zivilisatorischen Anlagen („Bädern“) zur Hilfe kommen. Die Kranken sind, bemerkt der Erzähler selbstironisch, vielmehr zum Sterben als zum Gesundwerden bereit: „Nein, wir wollen gerne sterben, später.“ (37)

Der Humor tritt ganz prägnant an der Stelle in den Vordergrund, wo der Erzähler zur Geschichte die denkbar größte Distanz einnimmt und sich fern von der subjektiven Stimmung des Pessimismus und des durch Krankheit und 
Langeweile herbeigeführten Missmuts hypostasiert. Dieser Erzähler lässt sich zugleich mit einem Autor identifizieren, der eine Selbstdarstellung nicht scheut:

Derjenige nun, welcher mir und den Gästen und dem allem zusah, dem gelangweilt essenden Hesse, den gelangweilt essenden Mitgästen, war nicht der Kurgast und Ischiatiker Hesse, sondern der alte, etwas gesellschaftsfeindliche Eremit und Sonderling Hesse, der alte Wanderer und Poet, der Freund der Schmetterlinge und Eidechsen, der alten Bücher und Religionen, jener Hesse, der sich der Welt entschlossen und kräftig gegenüberstellte und dem es ein tiefes Leid bereitete, wenn er sich von seiner Behörde einen Heimatschein ausstellen lassen oder auch nur den Zettel einer Volkszählung ausfüllen mußte. Dieser alte Hesse, dieses mir in der letzten Zeit etwas fremd gewordene und verlorengegangene Ich, war wieder da und schaute uns zu. (90)

Ist einmal der Schritt von dem am Geschehen teilhabenden, vom durch Pessimismus geplagten Ich-Erzähler zu einem objektiv beobachtenden Er-Erzähler getan, ist also der Perspektivenwechsel vollzogen, so wird auch die Vermittlung einer radikal neuen Stimmung möglich. Die „plötzlich aufgesprungene, ungeheure Lachlust“ (91) lässt nunmehr das eigene Handeln und das äußere Geschehen in einem komischen Licht erscheinen, und was zuvor „Langeweile und Trübsinn“ war, verwandelt sich in eine kaum zu zügelnde Heiterkeit. Sittliche Gesetze, zu der wohl auch die Gewohnheit der Klage und des Unmuts gehörten, werden überschritten, die Norm des schönen Betragens trotz inneren Schlechtbefindens wird der Unflätigkeit preisgegeben: Einem Gegenübersitzenden - der wie eine Figur „aus einem Roman von Turgenjew oder Thomas Mann“ aussieht - kann nunmehr schlicht und einfach vorgeworfen werfen, er sei ebenso unwirklich wie das ganze Milieu der Kurgäste. Krankheit und Langeweile, Missmut und Pessimismus können nicht mehr tragisch sein, alles löst sich im Lächerlichen und Heiteren auf:

Alles zusammen aber, Speisesaal und Welt, Kurgäste und Menschheit, schien mir, einen Augenblick lang, keineswegs entsetzlich und tragisch, sondern bloß ungeheuer lächerlich. Man brauchte ja nur zu lachen, so war der Bann durchstoßen, die Mechanik durchbrochen, so zogen Gott und die Vögel und Wolken durch unsern öden Saal, und wir waren nicht mehr trübe Gäste an der Kurtafel, sondern vergnügte Gäste Gottes an der bunten Tafel der Welt. (92) 
Das Lachen besiegt somit das Pessimistische, die Heiterkeit den Trübsinn. „Die Heiterkeit“, schrieb Hesse später, „hat die Kraftquellen in einem Grad von Kälte und Verzweiflung" (Hesse, Ausgewählte Briefe 75): Das Gleiche ist auch im Kurgast der Fall. Heiterkeit kann erlangt werden, sobald man, mittels der Gabe des Humors, seine eigene Verzweiflung überwindet. Erst dann ist das Lachen stärker als das Leiden.

Dem Kurgast, dieser Erzählung mit glänzendem Humor und tröstlicher Weisheit, die thematisch-stilistisch Jean Paul und vor allem seinem Katzenberger verpflichtet ist (Maurer 1968), folgte der Roman Der Steppenwolf (1927), in dem Depression, Pessimismus und suizidale Obsession mit aller Kraft zurückkehrten. Im „Tractat vom Steppenwolf“ ging Hesse sogar so weit, Selbstmord als eine Tat stärkerer Naturen zu deuten, die keine Pessimisten oder Nihilisten sein müssen, um diesen letzten Entschluss zu fassen - vielmehr sei der Selbstmord solcher starken Seelen ein Zeichen der Würde, der Willensfreiheit. Dennoch verzichtet der „Steppenwolf“ Harry Haller auf diesen letzten Schritt und begibt sich vielmehr auf den Weg psychischer Heilung. Allerdings kommt die rettende Hilfe von außen, dem ,weiblichen Alter Ego" Hermine oder dem Saxophonisten Pablo, ja, auch Rauschmittel kommen mit ins Spiel. Doch wäre es verfehlt, im „Magischen Theater“ ein Plädoyer für Betäubung als Gegenwehr gegen psychische Krankheiten zu sehen; vielmehr inszeniert Hesse einen psychischen Heilungsprozess, analog zu einer psychotherapeutischen Hypnose, bei der unbewusste, verdrängte psychische Inhalte und Traumata sozusagen an den Tag des Bewusstseins gefördert werden. Nicht zuletzt leistet das Lachen wiederum seinen Beitrag zur Heilung, insofern die „Unsterblichen“ (wie Mozart oder Goethe) in Harrys Traum ein lautes Gelächter anstimmen und ihm das Lachen als Heilkraft empfehlen. Den Spruch, dass Lachen gesund macht, hat Hesse hier gleichsam in poetische Dimensionen erhoben und psychologisch untermauert. Damit behält die Heiterkeit doch das letzte Wort, die seine Texte trotz aller depressiven Inhaltskomponenten zu heilenden Lektüren machen.

\section{Literaturverzeichnis}

Hesse, Hermann. Ausgewählte Briefe. Suhrkamp, 1974.

---. Meistererzählungen. Diogenes, 1977.

---. Gesammelte Werke in zwölf Bänden. Volker Michels (Hg.). Suhrkamp, 1987.

Limberg, Michael (Hrsg.). Hermann Hesse und die Psychoanalyse: „Kunst als Therapie“. 9. Internationales Hermann-Hesse-Kolloquium in Calw 1997. Bernhard Gengenbach, 1997. 
Lorenz, Martin. Musik und Nihilismus. Zur Relation von Kunst und Erkennen in der Philosophie Nietzsches. Königshausen \& Neumann, 2008.

Maurer, Warren R. „Jean Paul and Hermann Hesse: Katzenberger and Kurgast.“ Seminar, H. 4, Nr. 2, 1968, S. 113-28.

Meyer, Theo. Nietzsche. Kunstauffassung und Lebensbegriff. Francke, 1991.

Schmaus, Marion. Psychosomatik: literarische, philosophische und medizinische Geschichten zur Entstehung eines Diskurses (1778-1936). Niemeyer, 2009.

V. Szabó, László. Der Einfluss Friedrich Nietzsches auf Hermann Hesse. Formen des Nihilismus und seiner Überwindung bei Nietzsche und Hesse. Praesens, 2007.

---. „Hermann Hesses Konzept des Individuums.“ Vielheit und Einheit der Germanistik weltweit. Akten des XII. Internationalen Germanistenkongresses Warschau 2010. Grucza Franciszek et al. (Hg.). Peter Lang, 2012, S. 267-71.

---. „Taedium vitae. Zu Hermann Hesses Schopenhauer-Rezeption.“ Hermann Hesse und die Moderne. Diskurse zwischen Ästhetik, Ethik und Politik. Beiträge der internationalen Tagung aus Anlass des fünfzigsten Todestages des Dichters vom 2. bis 5. Mai 2012 am Germanistischen Institut der Universität Szeged. Detlef Haberland und Géza Horváth (Hg.). Praesens, 2013, S. 130-43. 


\section{“ICH WAR ALS NEUROTIKER ERKANNT...” ILLNESS, SUFFERING, AND PAIN IN HERMANN HESSE}

\begin{tabular}{c} 
Abstract \\
\hline László V. SZABÓ \\
Pannonische Universität Veszprém, Hungary \\
Egyetem u. 10, H-8201 Veszprém \\
Universität J. Selye, Slovakia \\
Bratislavská cesta 3322, 945 01 Komárno \\
vszabol@googlemail.com
\end{tabular}

The paper examines the interrelated topics of illness, suffering, and pain in several narrative texts by Hermann Hesse, such as Taedium vitae, Gertrud, A Guest at the Spa, and The Steppenwolf. The analysis focuses on the relation between illness, geniality, and art, partly biographical, partly based on contemporary discourses on (psychogenic) illness and healing, as they were prefigured in Nietzsche and Freud. The paper also reveals connections between Hesse's concept of illness as a psychogenic phenomenon and his understanding of art. Finally, psychologic and physiological processes of healing as described in A Guest at the Spa or The Steppenwolf are also considered.

Keywords: Illness, geniality, psychoanalysis, art, poetics, humor 


\title{
„ICH WAR ALS NEUROTIKER ERKANNT...“ BOLEST, PATNJA I BOL KOD HERMANNA HESSEA
}

\author{
Sažetak \\ László V. SZABÓ \\ Pannonische Universität Veszprém, Mađarska \\ Egyetem u. 10, H-8201 Veszprém \\ Universität J. Selye, Slovačka \\ Bratislavská cesta 3322, 94501 Komárno \\ vszabol@googlemail.com
}

Rad istražuje međusobno povezane teme bolesti, patnje i boli u nekoliko pripovjednih tekstova Hermanna Hessea, poput Taedium vitae, Gertrude, Gosta u toplicama i Stepskog vuka. Analiza se bavi odnosom bolesti, genijalnosti i umjetnosti, djelomično biografskim, a djelomično utemeljenim na suvremenim diskursima o (psihogenim) bolestima i ozdravljenju, kako ih donose Nietzsche i Freud. Isto tako, rad razotkriva poveznice između Hesseova koncepta bolesti kao psihogenog fenomena te njegova poimanja umjetnosti. U konačnici, rad razmatra psihološke i fiziološke procese ozdravljenja opisane u Gostu u toplicama i Stepskom vuku.

Ključne riječi: bolest, genijalnost, psihoanaliza, umjetnost, poetika, humor 